Check for updates

Cite this: Chem. Commun., 2017, 53, 11877

Received 19th September 2017 Accepted 11th October 2017

DOI: 10.1039/c7cc07310k

rsc.li/chemcomm

\section{Unprecedented rearrangement of diketopyrrolopyrroles leads to structurally unique chromophores $\dagger$}

\author{
Olena Vakuliuk, (D) ${ }^{a}$ Shota Ooi, (D) $\ddagger^{a}$ Irena Deperasińska, (DD ${ }^{b}$ \\ Olga Staszewska-Krajewska, (D) a Marzena Banasiewicz, (D) ${ }^{b}$ \\ Bolesław Kozankiewicz, (D) ${ }^{b}$ Oksana Danylyuk (D) ${ }^{c}$ and Daniel T. Gryko (D) *a
}

\begin{abstract}
Diketopyrrolopyrroles possessing thienyl, furyl and benzofuryl substituents undergo unprecedented skeletal rearrangement in the presence of trimethylsilyl bromide resulting in the formation of thieno[2,3-f]isoindole-5,8-diones and furo[2,3-f]isoindole-5,8-diones. These relatively small dyes possess favorable photophysical properties with the emission maxima within the range of 573-624 nm, large fluorescence quantum yields, moderate sensitivity of emission to solvent polarity and a HOMO-LUMO gap of ca. $1.8 \mathrm{eV}$.
\end{abstract}

Organic electronics and fluorescence imaging require heterocycles with $\pi$-extended conjugation. ${ }^{1}$ Rational design, aided by combinatorial and diversity-orientated approaches, provides invaluable routes toward novel functional materials. ${ }^{2}$ Nonetheless, certain sets of chromophores evade even the best designed retrosynthetic analysis and the serendipity factor tends to play a role in the discovery of new dyes. ${ }^{3}$ Depending on the targeted application, fluorophores possessing different combinations of properties are desirable. Small red-emitters are pivotal for applications in live fluorescence imaging. ${ }^{4}$

As a part of a larger project aimed at the synthesis of novel fluorophores, we investigated oxidative aromatic coupling of diketopyrrolopyrroles (DPPs, Fig. S1, ESI $\dagger$ ). ${ }^{5}$ We focused on iodine(III) reagents which efficiently promote such transformations, making them often the reagents of choice. ${ }^{6}$ During this study, we observed that subjecting 2,5-dimethyl-1,4-diketo-3,6-di(thien-3yl)pyrrolo[3,4-c]pyrrole (1) to hydroxy(tosyloxy)iodobenzene (Koser's reagent, HTIB) and TMSBr led to the formation of a purple compound as the sole isolable product (Scheme 1 and Table 1,

\footnotetext{
${ }^{a}$ Institute of Organic Chemistry, Polish Academy of Sciences, Kasprzaka 44/52, 01-224 Warsaw, Poland. E-mail: dtgryko@icho.edu.pl

${ }^{b}$ Institute of Physics, Polish Academy of Sciences, Al. Lotników 32/46, 02-668 Warsaw, Poland

${ }^{c}$ Institute of Physical Chemistry, Polish Academy of Sciences, Kasprzaka 44/52, 01-224 Warsaw, Poland

$\dagger$ Electronic supplementary information (ESI) available. CCDC 1574216. For ESI and crystallographic data in CIF or other electronic format see DOI: 10.1039/ c7cc07310k

\# Current address: Kyoto University, Department of Chemistry, Japan.
}
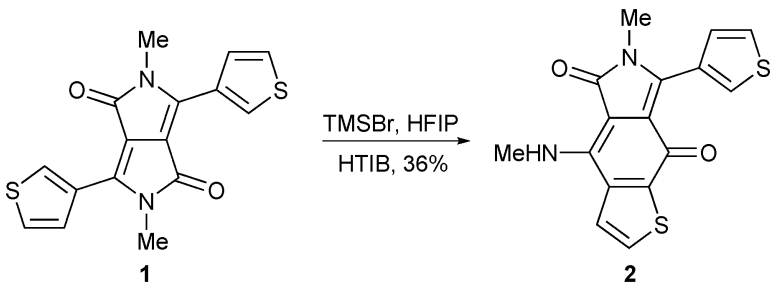

Scheme 1 The rearrangement of DPP 1 into thieno[2,3-f]isoindole-5,8dione 2 .

entry 1$)^{7}$ Surprisingly, while the exact mol. mass of a new compound turned out to be equal to the mol. mass of the substrate, the ${ }^{1} \mathrm{H}$ NMR data revealed the loss of symmetry in the product (Fig. S2, ESI $\dagger$ ). Such findings are consistent only with the rearrangement of the DPP 1.

NMR analysis provides structural information about the synthesized compound. The ${ }^{13} \mathrm{C}$ NMR spectrum shows two signals in the carbonyl region (171.1 ppm and $165.6 \mathrm{ppm}$ ) along with two signals in the aliphatic region $(32.8 \mathrm{ppm}$ and $28.7 \mathrm{ppm})$. Moreover, the ${ }^{1} \mathrm{H}$ NMR spectrum shows a singlet at $3.38 \mathrm{ppm}$ and a doublet at $3.42 \mathrm{ppm}$, which we ascribe as methyl groups. The difference in the chemical shifts as well as the multiplicity of the signals reconfirms the formation of the asymmetric product. The character of the proton signal at $8.82 \mathrm{ppm}\left({ }^{1} \mathrm{H}\right.$ NMR, broad quartet) reveals the opening of one $\gamma$-lactam ring and the presence of an $\mathrm{NH}-\mathrm{CH}_{3}$ moiety in the product. Moreover, COSY experiments support a $3+2$ spin system with one uncorrelated downfield signal. The reaction on one of the carbons adjacent to the sulfur atom can rationalize this finding. Finally, thorough analysis of ${ }^{1} \mathrm{H}^{13} \mathrm{C}$ HSQC and ${ }^{1} \mathrm{H}^{13} \mathrm{C}$ HMBC spectra revealed that the structure of 2 possesses the thieno[2,3-f] isoindole-5,8-dione core, which has so far never been described in the literature (Scheme 1). The final proof was obtained from single crystal X-ray analysis (Fig. S2 and S3, ESI†).

Encouraged by the unique structure of the prepared dye, we started a systematic study of the discovered transformation. The mixture of TMSBr and HTIB provides only moderate yields 
Table 1 Influence of the reaction conditions on the rearrangement of DPP 1 into dye $\mathbf{2}^{a}$

\begin{tabular}{lllll}
\hline Entry & Lewis acid & Additive, eq. & Solvent & Yield, $^{b} \%$ \\
\hline 1 & TMSBr & HTIB (2) & HFIP & 36 \\
2 & TMSBr & HTIB (0.05) & HFIP & 75 \\
3 & TMSBr & - & HFIP & 73 \\
4 & - & HTIB (2) & HFIP & 0 \\
5 & TMSBr & - & TFE $^{c}$ & 75 \\
6 & TMSBr & - & MeOH & 0 \\
7 & TMSBr $_{3}$ & - & DCM & 0 \\
8 & $\mathrm{BF}_{3} \cdot \mathrm{Et}_{2} \mathrm{O}$ & - & HFIP & 53 \\
9 & $\mathrm{BF}_{3} \cdot \mathrm{Et}_{2} \mathrm{O}^{d}$ & - & TFE $^{c}$ & 72
\end{tabular}

${ }^{a}$ Unless otherwise noted, reactions were performed with $\mathbf{1}(0.15 \mathrm{mmol})$, Lewis acid $(0.15 \mathrm{mmol})$, solvent $(1 \mathrm{ml}), 50{ }^{\circ} \mathrm{C}, 17 \mathrm{~h} .{ }^{b}$ Isolated yields are reported. ${ }^{c}$ Reaction was performed at $70{ }^{\circ} \mathrm{C} .{ }^{d} 2$ eq. of the Lewis acid was utilized.

of the expected product, strongly emphasizing the need for optimization of the reaction conditions (Table 1 , entry 1 ). In fact, the iodine(III) reagent does not support the formation of dye 2 product which suggests the non-oxidative character of this transformation. Rather, HTIB causes the decomposition of the desired product when added in excess (Table 1, entries 1-3). Conversely, the reaction does not proceed in the absence of TMSBr (Table 1, entry 4). We also elaborated the study of the discovered transformation to exhibit strong solvent dependence, which points to the importance of an acidic medium (Table 1, entries 3 and 5-7). In addition, improved solubility of the parent DPP aids a favorable outcome.

Aiming to increase the yield of the desired product, we tested a stronger Lewis acid, $\mathrm{BF}_{3} \cdot \mathrm{Et}_{2} \mathrm{O}$. Experimental investigations have proven the formation of dye 2 in up to $72 \%$ yield (Table 1, entries 8 and 9). It is noteworthy that the use of $\mathrm{BF}_{3} \cdot \mathrm{Et}_{2} \mathrm{O}$ requires a higher loading and elevated temperature to achieve a reasonable yield of the rearranged product. Other Lewis acids such as $\mathrm{POCl}_{3}$, TfOH, TFA or $\mathrm{BCl}_{3}$ do not mediate this rearrangement (Table S1, ESI $\dagger$ ).

By adopting conditions 2 and 5 (Table 1), we undertook the synthesis of analogues of heterocycle 2. Experimental findings showed a negligible effect of the alkyl substituents on the discovered transformation (Scheme 2). Notably, in this case the reaction medium plays an important role. We observed a significant improvement in the yield of dye 4 ( $60 \%$ versus $75 \%$ ) when HFIP was used in place of TFE. Variations in the solubility of the substrate in the tested solvents can probably rationalize this finding. Surprisingly, the transformation of DPP 5, bearing additional tert-butyl groups, does not proceed as expected in either TFE or HFIP. Unexpectedly, the presence of HTIB in a catalytic amount leads to significant improvement in the reaction outcome (Scheme 2).

2,5-Di(n-hexyl)-1,4-diketo-3,6-di(thien-2-yl)pyrrolo[3,4-c]pyrrole (7) affords the desired product 8 with a minuscule $6 \%$ yield even if the reaction is conducted for 3 days. The addition of Koser's reagent improves the reactivity of DPP 7, however, probably due to the oxidative character, this reagent induces the decomposition of the product or intermediates. Luckily, the replacement of TFE with HFIP and lowering the reaction temperature to $50{ }^{\circ} \mathrm{C}$ led to the desired product in $32 \%$ yield.

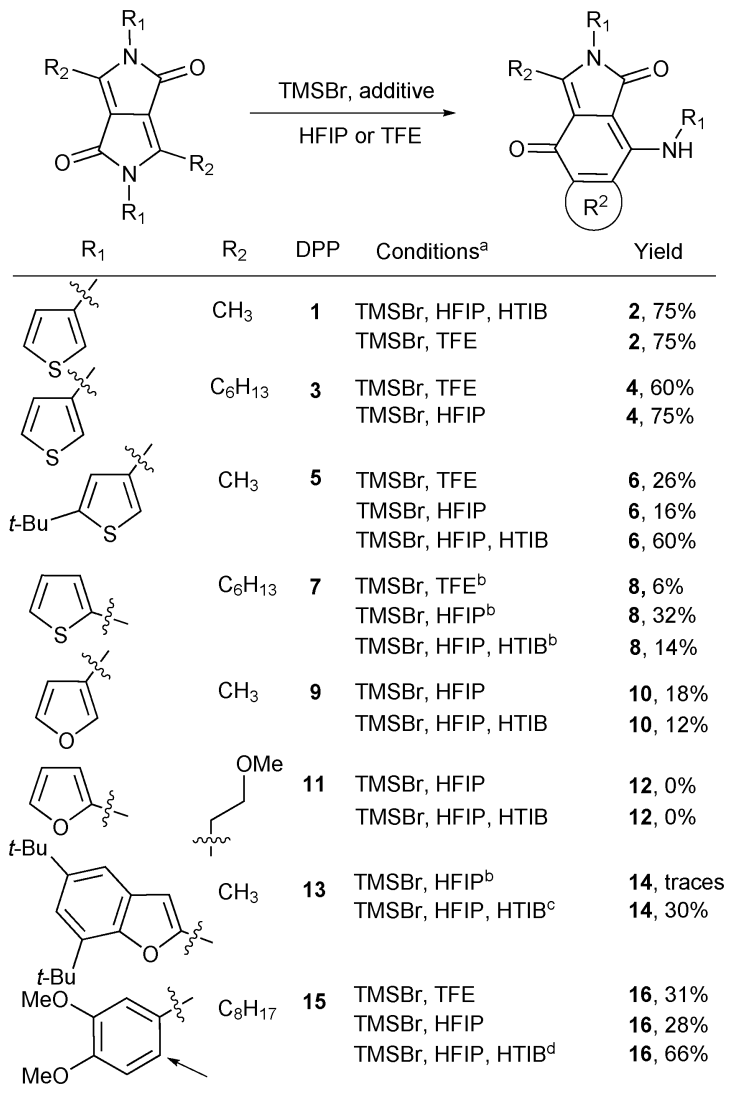

Scheme 2 Scope of the diketopyrrolopyrroles' rearrangement. ${ }^{a}$ Unless otherwise noted, reactions were performed with $\mathrm{TMSBr}(0.15 \mathrm{mmol})$, solvent ( $1 \mathrm{ml}$ ), for $17 \mathrm{~h}$ at $50{ }^{\circ} \mathrm{C}$ (for HFIP) or $70{ }^{\circ} \mathrm{C}$ (for TFE). Isolated yields are reported; ${ }^{b}$ reaction was conducted for 2 days; ${ }^{c}$ reaction was conducted for 3 days; ${ }^{d}$ reaction was performed with 2 eq. of Lewis acid.

Diketopyrrolopyrroles bearing 2-furyl and 3-furyl groups (9 and 11) display a lower reactivity compared to 1 and 7 , respectively. The desired product was obtained in low yield only for DPP 9. The attempts to improve the reaction outcome (HTIB, prolongation of the reaction time) did not bring about positive results. DPP 13, possessing a peripheral benzofuran moiety, displays poor reactivity as well. Fortunately, significant stability of the rearranged product allowed for the prolongation of the reaction time as well as utilization of the iodine(III) catalyst and, as a consequence, we could achieve a higher yield of 14. Under similar conditions, the rearrangement occurs for DPP 15 (Scheme 2), bearing 3,4-dimethoxyphenyl groups. $N$-Alkylated DPPs bearing less electron-rich aryl substituents do not undergo this rearrangement.

With the library of new dyes in hand, we conducted a series of photophysical studies, which reveal that the absorption bands corresponding to the lowest energy absorption are located in the 530-606 nm region (Table 2 and Fig. 1), displaying bathochromic shifts in comparison to the parent DPPs. Emission maxima are red-shifted to the orange-red part of the visible spectra. Compounds 2, 4 and 6 exhibit only small differences in absorption and emission maxima. Nevertheless, the type and position of the heteroatom influence $\lambda_{\mathrm{abs}}$ and $\lambda_{\mathrm{em}}$ significantly. Different charge distributions in the molecules 
Table 2 Photophysical properties of novel dyes measured in dichloromethane

\begin{tabular}{llllllll}
\hline & $\begin{array}{l}\lambda_{\mathrm{abs}} \\
{[\mathrm{nm}]}\end{array}$ & $\begin{array}{l}\lambda_{\mathrm{em}} \\
{[\mathrm{nm}]}\end{array}$ & $\begin{array}{l}\varepsilon_{\max } \\
{\left[\mathrm{M}^{-1} \mathrm{~cm}^{-1}\right]}\end{array}$ & $\Phi[\%]$ & $\begin{array}{l}\Delta S \\
{\left[\mathrm{~cm}^{-1}\right]}\end{array}$ & $\tau[\mathrm{ns}]$ & $\begin{array}{l}k_{\mathrm{r}} \\
{\left[10^{7} \mathrm{~s}^{-1}\right]}\end{array}$ \\
\hline $\mathbf{2}$ & 570 & 601 & 25500 & $48^{a}$ & 900 & 8.21 & 5.9 \\
$\mathbf{4}$ & 569 & 605 & 17200 & $49^{a}$ & 1100 & 7.78 & 6.3 \\
$\mathbf{6}$ & 571 & 602 & 17300 & $47^{a}$ & 900 & 6.45 & 7.3 \\
$\mathbf{8}$ & 582 & 619 & 16000 & $15^{a}$ & 1000 & 2.25 & 6.7 \\
$\mathbf{1 0}$ & 549 & 573 & 17500 & $13^{b}$ & 800 & 2.33 & 5.6 \\
$\mathbf{1 4}$ & 606 & 624 & 59400 & $20^{c}$ & 500 & 2.24 & 8.9 \\
$\mathbf{1 6}$ & 541 & 582 & 18200 & $57^{b}$ & 1300 & 7.25 & 7.9
\end{tabular}

${ }^{a}$ Calculated with respect to $\mathrm{Rh} 101$ in $\mathrm{MeOH} .{ }^{b}$ Calculated with respect to Rh6G in EtOH. ${ }^{c}$ Calculated with respect to SRh101 in EtOH.

can rationalize both the red-shift of the absorption and emission maxima when regioisomeric products $\mathbf{2}$ and $\mathbf{8}$ are superimposed and the blue-shift of $\lambda_{\mathrm{abs}}$ and $\lambda_{\mathrm{em}}$ for dyes possessing a furan unit (2 versus 10). Dye 14 possessing a $\pi$-expanded core is characterized by the most bathochromically shifted emission and absorption in the analyzed series.

Fluorescence responses for the majority of new dyes are rather high (Table 2). Fluorescence quantum yields $(\Phi)$ and $\lambda_{\mathrm{em}}$ are modestly influenced by the solvent polarity (Table S2, ESI $\dagger$ ). In the exemplary case of $2, \Phi$ changes from $56 \%$ in toluene to $28 \%$ in methanol (Table 3). This outcome is due to their moderately polarized electronic structures.

To rationalize these spectra, we performed additional quantum chemistry calculations for dye 2 , which concurred with experimental data (Fig. 2). According to our findings, the second excited $S_{2}$ state locates around $7000 \mathrm{~cm}^{-1}$ above the $S_{1}$, which means that the lowest excited $S_{1}$ state is well separated from the higher located, electronically excited states (Fig. 2 and Fig. S5 and Tables S3, S4, ESI $\dagger$ ). Moreover, the electronic transition $S_{0} \rightarrow S_{1}$ is of $\pi \rightarrow \pi^{*}$ character and corresponds to excitation from the HOMO to the LUMO level (Fig. 3).

In order to get more information about the reasons for smoothing the fluorescence spectra (lack any well resolved vibrational structure even at $5 \mathrm{~K}$ ) and the possible depopulation channels we simulated the vibrational structure of the fluorescence spectrum of 2 (Fig. S5, ESI $\dagger$ ). Calculation of the FranckCondon (FC) factors (Fig. S6, ESI $\dagger$ ) shows that the vibrational

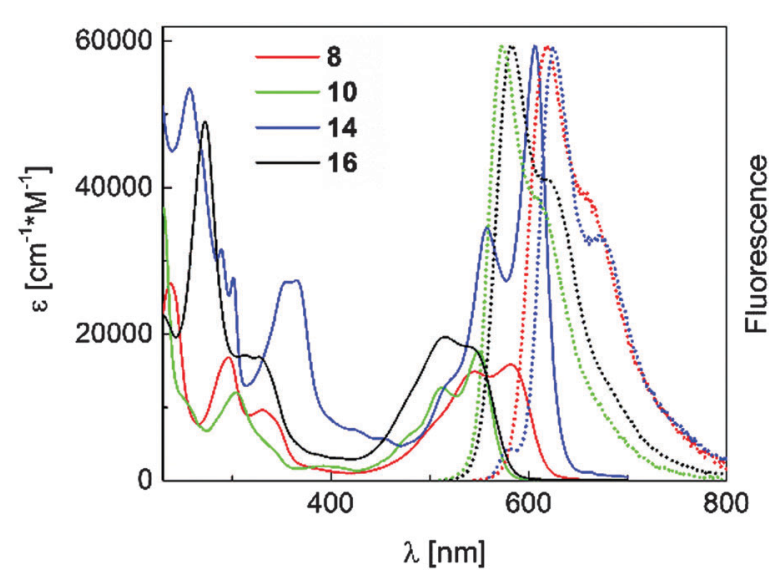

Fig. 1 Absorption and fluorescence spectra for 8, 10, 14 and 16 in DCM.
Table 3 Optical properties of $\mathbf{2}$ in various solvents

\begin{tabular}{lllll}
\hline Solvent & $\lambda_{\mathrm{abs}}[\mathrm{nm}]$ & $\lambda_{\mathrm{em}}[\mathrm{nm}]$ & $\Delta S\left[\mathrm{~cm}^{-1}\right]$ & $\Phi^{a}[\%]$ \\
\hline Hexane & 566 & 581 & 500 & - \\
Toluene & 573 & 599 & 800 & 56 \\
DCM & 570 & 601 & 900 & 48 \\
ACN & 566 & 603 & 1100 & 42 \\
MeOH & 565 & 617 & 1500 & 28 \\
${ }^{a}$ Calculated with respect to Rh101 in MeOH. & \\
\end{tabular}

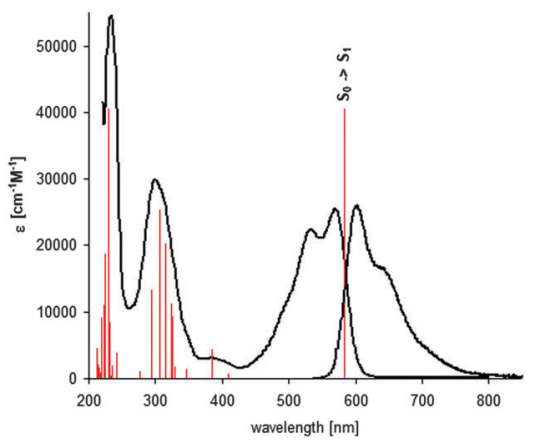

Fig. 2 Experimental absorption and fluorescence spectra of 2 in DCM. Calculated transition energies from the ground $S_{0}$ to the electronically excited states are given by vertical solid lines with the heights proportional to the oscillator strength of the transition (see Table S3, ESI $\dagger$ for details). Vibronic structure is smoothed by low frequency vibrations (for interpretation see Fig. S6, ESI†).

structure of the absorption and fluorescence spectra are obscured by the three low frequency vibrations: 37, 59 and $100 \mathrm{~cm}^{-1}$. These vibrations are characterized by high shift parameters $^{8}$ and contribute to the spectrum in their common combinations as well as in combination with other vibrations.

To elucidate the influence of the solvent polarity on the absorption and fluorescence spectra, we calculated dipole moments for 2 in the isolated form as well as in solution. The comparison of the HOMO and LUMO orbitals clearly depicts the charge shift in the excited $S_{1}$ state (Fig. 3). This shift causes an increase of the dipole moment (for $c a$. 1.5-2.2 D) and the bathochromic shift of both absorption and emission maxima in the polar solvents (Table S5, ESI $\dagger$ ).

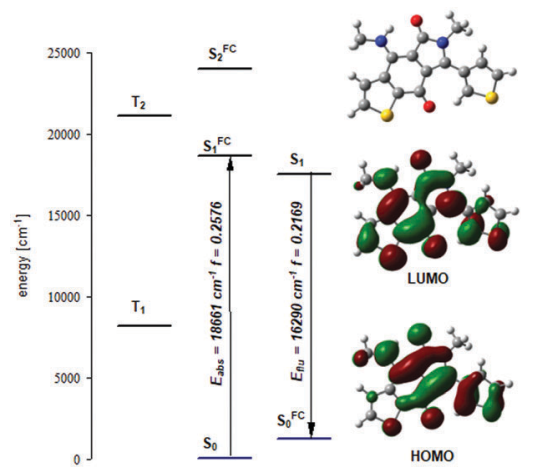

Fig. 3 Energy diagram with transition energies for absorption from the ground $S_{0}$ state to the $S_{1}^{F C}$ and fluorescence from the relaxed $S_{1}$ state to $\mathrm{S}_{0}^{\mathrm{FC}}$ for 2. On the right - the orbitals HOMO and LUMO of 2. Electronic configuration HOMO $\rightarrow$ LUMO describes the transition $\mathrm{S}_{0} \rightarrow \mathrm{S}_{1}$. 
Table 4 Redox potentials of the dyes 2, 4, 6, 8, 10, 14 and 16 measured in dichloromethane ${ }^{a}$

\begin{tabular}{llllll}
\hline Dye & $E_{\mathrm{ox}}^{\mathrm{pa}}, \mathrm{V}$ & $\mathrm{IP}, \mathrm{eV}$ & $E_{\text {red }}^{1 / 2}, \mathrm{~V}$ & $E_{\text {red }}^{\text {onset }}, \mathrm{V}$ & $\mathrm{EA}, \mathrm{eV}$ \\
\hline $\mathbf{2}$ & 0.90 & -5.1 & -1.10 & -1.02 & -3.3 \\
$\mathbf{4}$ & 0.96 & -5.1 & -1.15 & -1.06 & -3.3 \\
$\mathbf{6}$ & 0.86 & -5.1 & -1.17 & -1.07 & -3.3 \\
$\mathbf{8}$ & 0.92 & -5.1 & -1.15 & -1.03 & -3.3 \\
$\mathbf{1 0}$ & 0.94 & -5.2 & $-1.20^{b}$ & -1.05 & -3.3 \\
$\mathbf{1 4}$ & 0.89 & -5.1 & -0.93 & -0.81 & -3.5 \\
$\mathbf{1 6}$ & 0.80 & -5.0 & -1.28 & -1.19 & -3.2
\end{tabular}

${ }^{a}$ Measurement conditions: electrolyte $\left(\mathrm{NBu}_{4} \mathrm{ClO}_{4}, c=0.1 \mathrm{M}\right) ; \mathrm{DCM}_{\mathrm{dry}}$, potential sweep rate: $100 \mathrm{mV} \mathrm{s}^{-1}$, working electrode: glassy carbon (GC); auxiliary electrode: $\mathrm{Pt}$ wire; reference electrode: $\mathrm{Ag} / \mathrm{AgCl}$; all measurements were conducted at room temperature. ${ }^{b} E_{\text {red }}^{\mathrm{pc}}$ in V for $\mathbf{1 0 .}$

The performed calculations also provide information on the origin of the remaining non-radiative depopulation channel. We found that the excited $S_{1}$ state is located about $9500 \mathrm{~cm}^{-1}$ above the triplet $T_{1}$ and $2500 \mathrm{~cm}^{-1}$ below the $T_{2}$ states. Such an energetic situation precludes an efficient intersystem crossing pathway for the $S_{1} \rightarrow T_{1}$ depopulation. Thus, the main nonradiative depopulation channel of the $S_{1}$ state should proceed via internal conversion. This result concurs with our unsuccessful effort to detect phosphorescence emission at $5 \mathrm{~K}$.

Our calculations also indicate that 2 possesses a planar core with the peripheral thiophene ring rotated around a single bond by around $25^{\circ}$ with respect to the molecular plane (Table S4, ESI $\dagger$ ). Thus, new heterocycles are planar, structurally rigid fluorophores, the structure of which does not change substantially upon electronic excitation. As a consequence of the small conformational changes following the transition between $S_{0}$ and $S_{1}$ states in these compounds (additional computational data in Table S6, ESI $\dagger$ ) the Stokes' shifts are small.

To verify the potential use of the discovered dyes for optoelectronic applications we performed a series of cyclic voltammetry (CV) measurements, which depict the influence of structural changes on their susceptibility towards oxidation and reduction. In the majority of cases, the investigated molecules show reversible reduction and quasi-reversible oxidation waves (Table 4 and Fig. S5, ESI $\dagger$ ). The exception to the rule is observed only for $\mathbf{1 0}$ (irreversible oxidation). It is noteworthy that no changes were perceived with increasing number of redox cycles and the direction of the CV-measurement.

We calculated both ionization potential (IP) and electron affinity (EA) values from the corresponding onset potentials. The results clearly indicate that peripheral substituents, as well as the electronic nature of the aromatic rings participating in the rearrangement, have no significant influence on the ionic potential $(-5.1 \pm 0.1 \mathrm{eV})$ or on the electron affinity $(-3.3 \pm 0.2 \mathrm{eV})$.

Herein, we have introduced structurally unique dyes possessing an isoindoledione core, linearly fused with a thiophene, furan, or benzene ring. We have demonstrated that diketopyrrolopyrroles possessing moderately electron-rich aryl substituents undergo heretofore unknown rearrangements initiated by mild Lewis acids.
Strong emission of red light combined with straightforward synthesis, relatively small molecular mass, and potential chemical reactivity may open doors for these dyes for applications in fluorescence microscopy (bioimaging).

The financial support of the Polish National Science Centre (MAESTRO-2012/06/A/ST5/00216), Foundation for Polish Science (TEAM/2016-3/22) and the Global Research Laboratory Program (2014K1A1A2064569) through the National Research Foundation (NRF) funded by the Ministry of Science, ICT \& Future Planning (Korea) is cordially acknowledged. Theoretical calculations were performed at the Interdisciplinary Center of Mathematical and Computer Modeling (ICM) of the Warsaw University (Poland) under the computational grant No. G-32-10. S. O. acknowledges JSPS fellowships.

\section{Conflicts of interest}

There are no conflicts to declare.

\section{Notes and references}

1 For the selected reviews, see: (a) H. Yao, L. Ye, H. Zhang, S. Li, S. Zhang and J. Hou, Chem. Rev., 2016, 116, 7397; (b) M. Mas-Torrent and C. Rovira, Chem. Rev., 2011, 111, 4833; (c) Y. Tao, Ch. Yang and J. Qin, Chem. Soc. Rev., 2011, 40, 2943; (d) T. Tanaka and A. Osuka, Chem. Rev., 2017, 117, 2584; (e) C. Preihs, J. F. Arambula, D. Magda, H. Jeong, D. Yoo, J. Cheon, Z. H. Siddik and J. L. Sessler, Inorg. Chem., 2013, 52, 12184.

2 (a) A. Narita, X.-Y. Wang, X. Feng and K. Müllen, Chem. Soc. Rev., 2015, 44, 6616; (b) M. Stępień, E. Gońka, M. Żyła and N. Sprutta, Chem. Rev., 2017, 117, 3479; (c) W. Chen, C.-L. Chen, Z. Zhang, Y.-A. Chen, W.-C. Chao, J. Su, H. Tian and P.-T. Chou, J. Am. Chem. Soc., 2017, 139, 1636; (d) S. L. Broman, A. U. Petersen, C. G. Tortzen, J. Vibenholt, A. Bond and M. B. Nielsen, Org. Lett., 2012, 14, 318.

3 Incidental discoveries of new fluorophores are as commonly known as they were 150 years ago: (a) A. von Bayer, Chem. Ber., 1871, 5, 255; (b) M. Ishida, P. Kim, J. Choi, J. Yoon, D. Kim and J. L. Sessler, Chem. Commun., 2013, 49, 6950; (c) A. Osuka and H. Shimidzu, Angew. Chem., Int. Ed. Engl., 1997, 36, 135; (d) A. Janiga, E. GłodkowskaMrówka, T. Stokłosa and D. T. Gryko, Asian J. Org. Chem., 2013, 2, 411.

4 (a) H.-Y. Ahn, K. E. Fairfull-Smith, B. J. Morrow, V. Lussini, B. Kim, M. V. Bondar, S. E. Bottle and K. D. Belfield, J. Am. Chem. Soc., 2012, 134, 4721; (b) A. N. Butkevich, G. Y. Mitronova, S. C. Sidenstein, J. L. Klocke, D. Kamin, D. N. H. Meineke, E. D'Este, P.-T. Kraemer, J. G. Danzl, V. N. Belov and S. W. Hell, Angew. Chem., Int. Ed. Engl., 2016, 55, 3290; (c) W. Xu, Z. Zeng, J.-H. Jiang, Y.-T. Chang and L. Yuan, Angew. Chem., Int. Ed., 2016, 55, 13568; (d) C. Allain, F. Schmidt, R. Lartia, G. Bordeau, C. Fiorini-Debuisschert, F. Charra, P. Tauc and M. P. Teulade-Fichou, ChemBioChem, 2007, 8, 424; (e) D. Kim, H. Moon, S. H. Baik, S. Singha, Y. W. Jun, T. Wang, K. H. Kim, B. S. Park, J. Jung, I. Mook-Jung and K. H. Ahn, J. Am. Chem. Soc., 2015, 137, 6781.

5 (a) M. Grzybowski and D. T. Gryko, Adv. Opt. Mater., 2015, 3, 280; (b) E. H. G. Zadeh, M. V. Bondar, I. A. Mikhailov and K. D. Belfield, J. Phys. Chem. C, 2015, 119, 8864.

6 (a) Y. Kita and T. Dohi, Chem. Rec., 2015, 15, 886; (b) V. V. Zhdankin, ARKIVOC, 2009, 1-62; (c) Y. Kita, H. Tohma, K. Hatanaka, T. Takada, S. Fujita, S. Mitoh, H. Sakurai and S. Oka, J. Am. Chem. Soc., 1994, 116, 3684.

7 Y. Kita, K. Morimoto, M. Ito, C. Ogawa, A. Goto and T. Dohi, J. Am. Chem. Soc., 2009, 131, 1668.

8 V. Barone, J. Bloino, M. Biczysko and F. Santoro, J. Chem. Theory Comput., 2009, 5, 540-554. 